University of Nebraska - Lincoln

DigitalCommons@University of Nebraska - Lincoln

10-10-1997

\title{
Observation of laser satellites in a plasma produced by a femtosecond laser pulse
}

\author{
S.A. Pikuz \\ Optical Science, Ann Arbor, Michigan, USA \\ Anatoly Maksimchuk \\ University of Michigan, tolya@umich.edu \\ Donald Umstadter \\ University of Nebraska-Lincoln, donald.umstadter@unl.edu \\ M. Nantel \\ Center for Ultrafast Optical Science, Ann Arbor, Michigan, USA
}

P. N. Lebedev Physical Institute Russian Academy of Sciences, Moscow, Russia; Center for Ultrafast

I. Yu. Skobelev

Atomic Spectroscopy Data Center, All-Russia Scientific-Research Institute of Physicotechnical and Radio Electronic Measurements, Mendeleevo, Moscow Region, Russia

See next page for additional authors

Follow this and additional works at: https://digitalcommons.unl.edu/physicsumstadter

Part of the Physics Commons

Pikuz, S.A.; Maksimchuk, Anatoly; Umstadter, Donald; Nantel, M.; Skobelev, I. Yu.; Faenov, A. Ya.; and Osterheld, A., "Observation of laser satellites in a plasma produced by a femtosecond laser pulse" (1997). Donald Umstadter Publications. 43.

https://digitalcommons.unl.edu/physicsumstadter/43

This Article is brought to you for free and open access by the Research Papers in Physics and Astronomy at DigitalCommons@University of Nebraska - Lincoln. It has been accepted for inclusion in Donald Umstadter Publications by an authorized administrator of DigitalCommons@University of Nebraska - Lincoln. 


\section{Authors}

S.A. Pikuz, Anatoly Maksimchuk, Donald Umstadter, M. Nantel, I. Yu. Skobelev, A. Ya. Faenov, and A. Osterheld 


\title{
Observation of laser satellites in a plasma produced by a femtosecond laser pulse
}

\author{
S. A. Pikuz \\ P. N. Lebedev Physical Institute Russian Academy of Sciences, 118927 Moscow, Russia; \\ Center for Ultrafast Optical Science, Ann Arbor, Michigan, USA
}

A. Maksimchuk, D. Umstadter, and M. Nantel

Center for Ultrafast Optical Science, Ann Arbor, Michigan, USA

I. Yu. Skobelev and A. Ya. Faenov

Atomic Spectroscopy Data Center, All-Russia Scientific-Research Institute of Physicotechnical and Radio Electronic Measurements, 141570 Mendeleevo, Moscow Region, Russia

\author{
A. Osterheld \\ Lawrence Livermore National Laboratory, University of California, Livermore, CA 94550, \\ USA
}

(Submitted 2 September 1997)

Pis'ma Zh. Éksp. Teor. Fiz. 66, No. 7, 454-459 (10 October 1997)

Laser satellites are detected in the emission spectra of magnesium and aluminum plasmas produced by femtosecond laser pulses. This is made possible by the realization of picosecond time resolution in a highluminosity x-ray spectrograph with a spherically curved mica crystal. The temporal characteristics of these newly recorded spectral lines show unequivocally that they are formed as a result of nonlinear processes. (C) 1997 American Institute of Physics.

[S0021-3640(97)00219-3]

PACS numbers: $52.50 . \mathrm{Jm}, 52.25 . \mathrm{Nr}$

Strong oscillating electric and magnetic fields can greatly influence the emission characteristics of ions. Vinogradov and Yukov ${ }^{1}$ have shown theoretically that new spectral lines, which they called laser satellites, should appear in the emission spectrum of an ion placed in a strong laser field. The laser satellites are due to a nonlinear interaction of the radiation with the excited states $B^{*}$ of the ions, as a result of which the laser photons $\hbar \omega_{\text {las }}$ are converted into photons $\hbar \omega^{\prime}$ and $\hbar \omega^{\prime \prime}$ with the frequencies $\omega^{\prime}=\omega_{B} *_{B}+\omega_{\text {las }}$ and $\omega^{\prime \prime}=\omega_{B * B}-\omega_{\text {las }}$, where $\hbar \omega_{B *_{B}}$ is the difference of the energies of the ion in the states $B^{*}$ and $B$ (see Fig. 1). These nonlinear processes are possible if the states $B^{*}$ and $B$ of the ion possess the same parity. This means that the transition $B^{*} \rightarrow B$ itself is optically forbidden.

Thus a laser field with frequency $\omega_{\text {las }}$ should produce two new spectral lines around each parity-forbidden transition $B^{*} \rightarrow B$ of the ion. These lines have frequencies $\omega_{B *_{B}} \pm \omega_{\text {las }}$ and virtually the same intensities. ${ }^{1}$ It should be noted that if the ion $B$ is multiply charged, then, first, the wavelengths of the emitted laser satellites can lie in the 


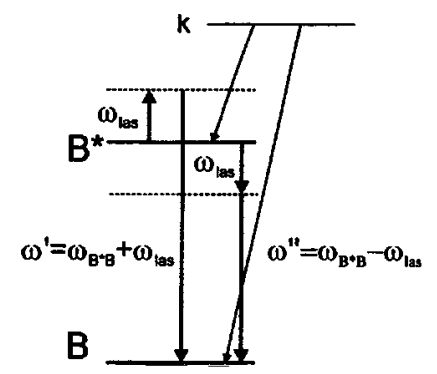

FIG. 1. Scheme of the nonlinear interaction of laser photons with multiply charged ions.

$\mathrm{x}$-ray region of the spectrum and, second, the probabilities of the nonlinear processes become quite high only in ultrahigh laser fields (see below).

Despite the fact that the laser x-ray satellites were predicted more than 20 years ago, they had not been observed until very recently. This is because their experimental detection requires: 1) an intense laser pulse with flux density $\left.\geqslant 10^{16} \mathrm{~W} / \mathrm{cm}^{2}, 2\right)$ an $\mathrm{x}$-ray spectrograph with a very good spectral resolution $\lambda / \Delta \lambda \geqslant 5000$, and 3) the capability of recording the emission spectrum of a plasma only during the interaction with the laser pulse. It is very difficult to satisfy these conditions in the same experiment. For example, it is quite easy to achieve high laser radiation flux densities $q>10^{17} \mathrm{~W} / \mathrm{cm}^{2}$ with a subpicosecond pulse, but in this case it is very difficult (even now) to obtain subpicosecond time resolution of the x-ray spectrograph. In the opposite case of a quite long laser pulse, laser satellites can be detected even without time resolution (time resolution can actually be replaced by good spatial resolution, since in this case there is enough time during the laser pulse for the plasma to leave the observation region), but this requires quite unique laser setups. We note that recently we were the first to observe ${ }^{2,3}$ laser satellites in the spectra of a titanium plasma by using one such setup - "Janus" — at the Lawrence Livermore National Laboratory (USA). This setup made it possible to obtain a laser radiation flux density $\approx 10^{17} \mathrm{~W} / \mathrm{cm}^{2}$ with pulse duration $\approx 120 \mathrm{ps}$. Somewhat later, laser satellites were also observed in the VUV range. ${ }^{4}$

In the present work we recorded laser satellites in the emission spectra of magnesium and aluminum plasmas produced by femtosecond laser pulses. This was made possible by the realization of picosecond time resolution in a high-luminosity $\mathrm{x}$-ray spectrograph with a spherically curved mica crystal. The temporal characteristics of the new spectral lines show unequivocally that the lines are formed as a result of the nonlinear processes discussed above.

\section{EXPERIMENTAL ARRANGEMENT}

The experiments were performed at the Center for Ultrafast Optical Science at the University of Michigan.

The x-ray spectra were excited by irradiating solid targets with a $400 \mathrm{fs}$ laser pulse $\mathrm{s}^{5}$ with energy up to $2 \mathrm{~J}$ at the fundamental frequency $(\lambda=1.053 \mu \mathrm{m})$. A spherically curved mica crystal $(R=186 \mathrm{~mm})$ focused the plasma radiation onto the photocathode of a 


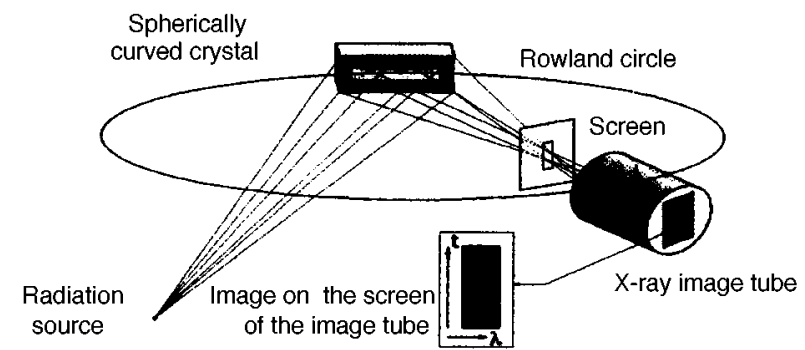

FIG. 2. Experimental arrangement for recording time-resolved x-ray spectra of a plasma.

Kentec x-ray camera in a slitless arrangement of a FSSR-1D spectrograph (see Fig. 2). ${ }^{6}$ The spectral resolution of the system in the dynamic regime was not worse than $\lambda / \Delta \lambda \approx 5000$. The time resolution at the maximum scan rate was equal to 5-7 ps. The image from the camera screen was intensified with a multichannel plate light amplifier and recorded with a 12-bit CCD camera; this made possible wavelength referencing of the images obtained in different shots.

\section{RESULTS AND DISCUSSION}

In the present experiment the relative arrangement of the plasma, the spherical crystal, and the detection system was chosen so as to record the emission spectrum in the ranges 9.15-9.35 $\AA$ (in second-order reflection from the mica crystal) and 6.1-6.23 $\AA$ (in third-order reflection). In the case of a magnesium plasma this made it possible to observe the resonance and intercombination lines of the He-like ion Mg XI and their dielectronic satellites and, in the case of an aluminum plasma, the $1 s 5 p{ }^{1} P_{1}-1 s^{2}{ }^{1} S_{0}$ line of the He-like ion Al XII. Laser satellites due to the interaction of laser photons with the $1 s 2 s{ }^{1} S_{0}$ state of the $\mathrm{Mg}$ XI ion and the $1 s 5 s{ }^{1} S_{0}$ and $1 s 5 d{ }^{1} D_{2}$ states of the Al XII ion should also fall into the indicated detection ranges.

In Ref. 1 it was shown that the intensity $P$ of laser satellites can be estimated according to the equation

$$
P\left(\mathrm{~W} / \mathrm{cm}^{3}\right)=\omega^{\prime} / \omega_{\text {las }} N_{B *} q_{\text {las }} \sigma,
$$

where $q_{\text {las }}\left(\mathrm{W} / \mathrm{cm}^{2}\right)$ is the laser radiation flux density, $N_{B^{*}}\left(\mathrm{~cm}^{-3}\right)$ is the population of the excited state $B^{*}$ of the ion, the frequencies are $\omega^{\prime}=\omega_{B * B^{*}} \pm \omega_{\text {las }}$, and $\sigma$ is the Raman scattering cross section, which can be estimated in the approximation of one virtual level according to the formula

$$
\sigma=\sigma_{T}\left[\omega_{\text {las }} \omega^{\prime 3}\left(\omega_{k B}+\omega_{k B}\right)^{2} f_{B k} f_{B * k}\right] /\left\{4\left(\omega_{k B} *-\omega_{\text {las }}\right)^{2}\left(\omega_{k B}+\omega_{\text {las }}\right)^{2} \omega_{k B} \omega_{k B} *\right\},
$$

where $\sigma_{T}=(8 \pi / 3)\left(e^{2} / m c^{2}\right)^{2}$ is the Thomson cross section and $f_{i j}$ is the oscillator strength for the transition $i \rightarrow j$.

Equation (1) can be rewritten, introducing the probability $A_{\text {las }}\left(\omega^{\prime}\right)$ of induced transitions, as

$$
P=\hbar \omega^{\prime} N_{B} A_{\text {las }}\left(\omega^{\prime}\right),
$$


where $A_{\text {las }}\left(\omega^{\prime}\right)=q_{\text {las }} \sigma / \hbar \omega_{\text {las }}$. When the energy $\hbar \omega^{\prime}$ of the emitted photon falls in the x-ray range, the probabilities $A_{\text {las }}\left(\omega_{B *_{B}}+\omega_{\text {las }}\right)$ and $A_{\text {las }}\left(\omega_{B * B}-\omega_{\text {las }}\right)$ are practically identical and therefore both laser satellites should have approximately equal intensities. One can see from Eqs. (2) and (3) that the most intense laser satellites are obtained if the state $B^{*}$ possesses the same multiplicity as the lower level $B$ of the transition, since then the oscillator strengths $f_{B k}$ and $f_{B * k}$ will correspond to optically allowed transitions.

The interaction of a laser photon $\hbar \omega_{\text {las }}=9524 \mathrm{~cm}^{-1}$ with the $1 s 2 s{ }^{1} S_{0}$ state of a $\mathrm{Mg}$ $\mathrm{XI}$ ion should give two satellite lines with wavelengths $\lambda_{s 1} \approx 9.2178 \AA$ and $\lambda_{s 2} \approx 9.2341$ $\AA$, since according to the calculations performed in Ref. 7, the energy of the $1 s 2 s{ }^{1} S_{0}$ level equals $1.0839 \times 10^{7} \mathrm{~cm}^{-1}$. The probabilities $A_{\text {las }}\left(\omega^{\prime}\right)$ of induced transitions can be estimated from Eqs. (2) and (3), taking the $1 s 2 p{ }^{1} P_{1}$ state as the level $k$ (see Fig. 1). Using the data given in Ref. 7 for the energies of the levels and oscillator strengths, we obtain $A_{\text {las }}\left(\lambda_{S 1, S 2}\right) \approx 1.5 \times 10^{13}\left(q_{\text {las }} / 10^{17} \mathrm{~W} / \mathrm{cm}^{2}\right) \mathrm{s}^{-1}$. For $q_{\text {las }}>10^{16} \mathrm{~W} / \mathrm{cm}^{2}$ the probabilities $A_{\text {las }}\left(\lambda_{S 1, S 2}\right)$ are higher than the probabilities of collisional de-excitation of the $1 s 2 s{ }^{1} S_{0}$ level and the intensities of the laser satellites can be estimated on the basis of a coronal model. Using the excitation rates of the $1 s 2 p{ }^{1} P_{1}$ and $1 s 2 s{ }^{1} S_{0}$ levels calculated in Ref. 8, we obtain for the ratio of the intensities of the laser satellites to the intensity of the resonance line

$$
I\left(\lambda_{S 1, S 2}\right) / I_{R}=1 / 2\left[\left\langle\nu \sigma\left(1 s^{2}-1 s 2 s{ }^{1} S_{0}\right)\right\rangle\left\langle\nu \sigma\left(1 s^{2}-1 s 2 p{ }^{1} P_{1}\right)\right\rangle\right] \approx 0.15,
$$

where $I_{R}$ is the intensity of the resonance line of the Mg XI ion and $\langle\nu \sigma\rangle$ are the rates of excitation of the $1 s 2 s{ }^{1} S_{0}$ and $1 s 2 p{ }^{1} P_{1}$ levels by electron impact from the $1 s 2 s{ }^{1} S_{0}$ ground state. It is clear from Eq. (4) that the laser satellites are sufficiently intense to be detected experimentally. However, it should be underscored that if the laser satellites are emitted only during the time $\tau_{\text {las }}$ of the interaction with the laser pulse, then the standard spectral lines are emitted during the plasma lifetime $\tau_{\text {plasma }}$, which is determined by gasdynamic processes and, as a rule, is much longer (by 10-100 times) than the laser pulse. In consequence, when spectra are recorded without time resolution the average intensity of the laser satellites will be determined by the expression

$\left\langle I_{s 2} / I_{R}\right\rangle=\left\langle I_{s 1} / I_{R}\right\rangle=1 / 2\left[\left\langle\nu \sigma\left(1 s^{2}-1 s 2 s{ }^{1} S_{0}\right)\right\rangle /\left\langle\nu \sigma\left(1 s^{2}-1 s 2 p{ }^{1} P_{1}\right)\right\rangle \tau_{\text {las }} / \tau_{\text {plasma }}\right]$

which for the case of the present experiment gives $\approx 0.01$, which does not exceed the noise level of the spectrograms.

The experimental results presented in Fig. 3 illustrate what we have said above. The time-integrated emission spectrum of a magnesium plasma in the range 9.15-9.35 $\AA$ is displayed in Fig. 3a, and the region of the spectrum near the intercombination line $1 s 2 p{ }^{3} P_{1}-1 s^{2}{ }^{1} S_{0}$ corresponding to two different times - during the laser pulse (curve 1) and immediately after the pulse (curve 2 ) - is shown in Fig. 3b. Figure $3 \mathrm{~b}$ also shows a model spectrum (curve 3) constructed in accordance with expression (6). It is seen from Fig. 3a that in the time-integrated spectrum the laser satellites are indeed indistinguishable against the background noise in the spectrogram. The laser satellites are similarly absent after the heating pulse ends (see curve 2 in Fig. 3b), while during the laser pulse (curve 1 in Fig. 3b) their position and intensity agree very well with the theoretical estimates (curve 3 in Fig. 3b). 

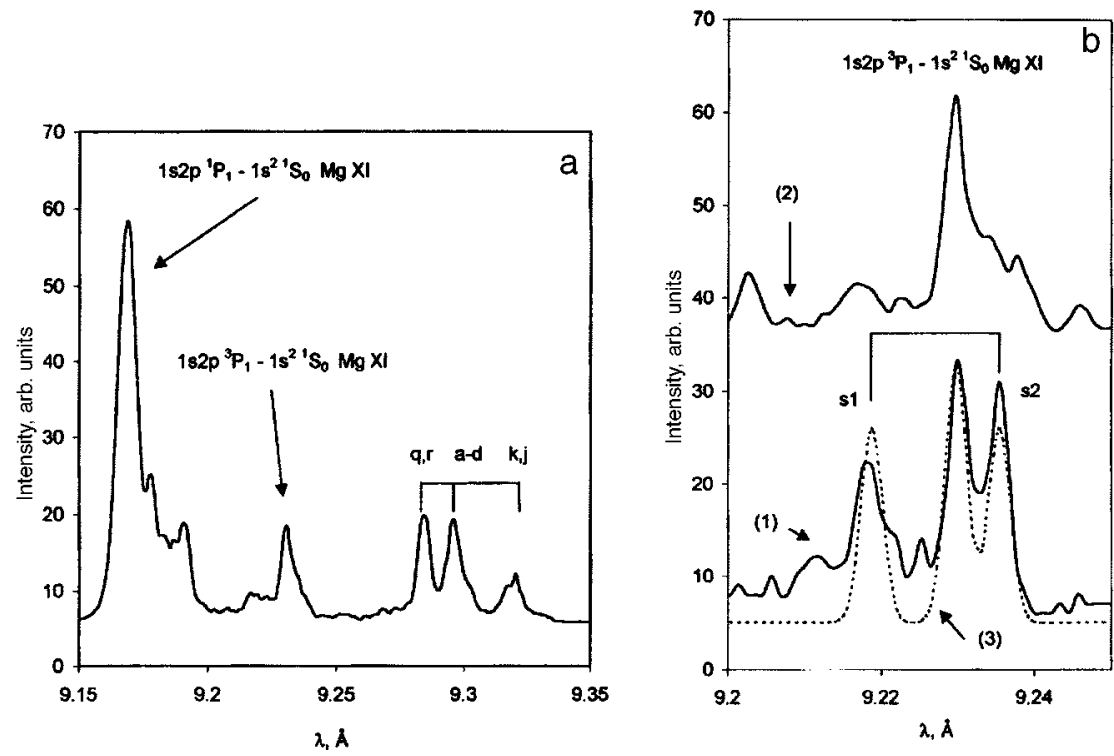

FIG. 3. a) Time-integrated emission spectrum of a femtosecond magnesium laser plasma; b) radiation from a magnesium plasma during the laser pulse (1) and immediately after the pulse (2). Curve 3 is the result of a theoretical modeling.

Similar results are also obtained for the laser satellites of the $1 s 5 p{ }^{1} P_{1}-1 s^{2}{ }^{1} S_{0}$ line of the He-like ion Al XII (see Fig. 4). In this case it is possible to observe four laser satellites, which are due to the interaction of the laser photons with the $1 s 5 d{ }^{1} D_{2}$ state (lines a,c in Fig. 4) and the $1 s 5 s{ }^{1} S_{0}$ state (lines b,d in Fig. 4). Using once again the coronal model and the estimates given in Ref. 9 for the excitation rates of the transitions $1 s^{2} \rightarrow 1 s 5 l{ }^{1} L(l=s, p, d)$, we find that at the plasma temperature $T_{e} \approx 200 \mathrm{eV}$ the intensities of these laser satellites should be $\approx 0.37$ times the intensity of the allowed transition $1 s 5 p{ }^{1} P_{1}-1 s^{2}{ }^{1} S_{0}$. The model spectrum constructed in this manner (curve 3 in Fig. 4) corresponds to the experimental spectrum emitted during the laser pulse (curve 1 in Fig. 4). We underscore also that no lines are observed at the locations of the laser satellites in the spectrum emitted by the plasma immediately after the laser pulse (curve 2 in Fig. 4).

Since $E_{B} *\left[\mathrm{~cm}^{-1}\right]=10^{8}\left(1 / \lambda_{s 1}[\AA]+1 / \lambda_{s 2}[\AA] / 2\right.$, the detection of laser satellites provides a direct method for measuring the excitation energies of the metastable levels of ions (see Table I). It should be underscored, however, that in the presence of a strong laser field the levels will be shifted with respect to the levels of an isolated ion on account of the quadratic Stark effect. For example, for the $1 s 2 s{ }^{1} S_{0}$ level of the ion Mg XI a simple estimate ${ }^{10}$ gives $\Delta E=-6.6 \times 10^{4}\left(q_{\text {las }} / 10^{17}\left[\mathrm{~W} / \mathrm{cm}^{2}\right]\right) \mathrm{cm}^{-1}$. For $q_{\text {las }}>10^{6} \mathrm{~W} / \mathrm{cm}^{2}$ the shift is appreciably larger than the measurement error, and if the position of the level of an isolated ion is known, then it can be used to estimate the laser radiation flux density in the plasma. In our case, as follows from a comparison of the experimental results with the calculation performed in Ref. 7 for an isolated ion by the relativistic perturbation method allowing for QED corrections, the flux density did not exceed $q_{\text {las }}=10^{16} \mathrm{~W} / \mathrm{cm}^{2}$. 


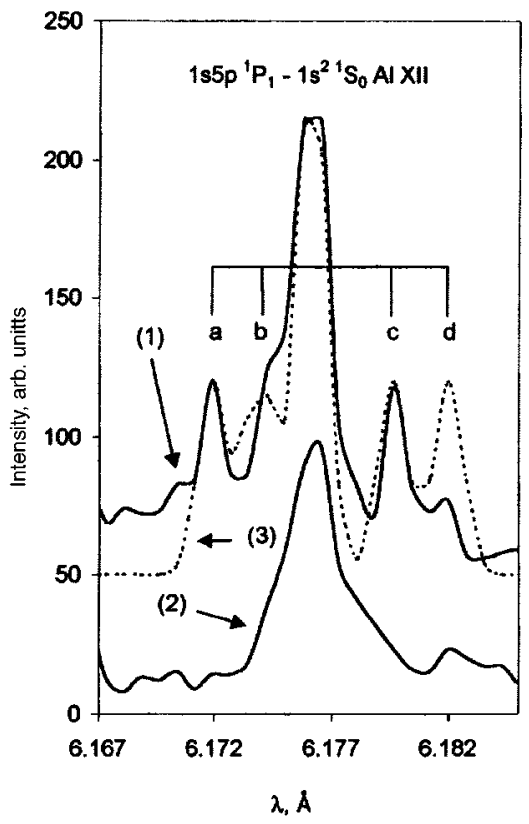

FIG. 4. a) Emission from an aluminum plasma during the laser pulse (1) and immediately after the pulse (2). Curve 3 is the result of a theoretical modeling.

In summary, the detection of $\mathrm{x}$-ray emission from a plasma with picosecond time resolution achieved in the present work made it possible to observe laser satellites excited in a femtosecond laser plasma. The fact that the radiation in the observed spectral lines is emitted only during the interaction with the heating laser pulse indicates unequivocally that the lines are produced by the nonlinear interaction of laser photons with the excited states of multiply charged manganese and aluminum ions. Improvement of the time

TABLE I. Energies of metastable states of the He-like ions Mg XI and Al XII.

\begin{tabular}{|c|c|c|c|c|c|}
\hline \multirow[b]{2}{*}{ Ion } & \multirow[b]{2}{*}{ Level } & \multicolumn{2}{|l|}{ Laser satellites } & \multicolumn{2}{|c|}{ Energy, $\mathrm{cm}^{-1}$} \\
\hline & & Transition & $\lambda_{\text {exp }}, \AA$ & experiment & theory $^{7}$ \\
\hline Mg XI & $1 s 2 s{ }^{1} S_{0}$ & $\left\{\begin{array}{l}\left(1 s 2 s{ }^{1} S_{0}-1 s^{2}{ }^{1} S_{0}+\omega_{\text {las }}\right. \\
1 s 2 s{ }^{1} S_{0}-1 s^{2}{ }^{1} S_{0}-\omega_{\text {las }}\end{array}\right.$ & $\begin{array}{l}9.2199(11) \\
9.2366(11)\end{array}$ & $1.0836(3) \times 10^{7}$ & $1.0839 \times 10^{7}$ \\
\hline Al XII & $1 s 5 s{ }^{1} S_{0}$ & $\left\{\begin{array}{l}1 s 5 s{ }^{1} S_{0}-1 s^{2}{ }^{1} S_{0}+\omega_{\mathrm{las}} \\
1 s 5 s{ }^{1} S_{0}-1 s^{2}{ }^{1} S_{0}-\omega_{\mathrm{las}}\end{array}\right.$ & $\begin{array}{l}6.1732(8) \\
6.1813(8)\end{array}$ & $1.6188(3) \times 10^{7}$ & $1.6188 \times 10^{7}$ \\
\hline $\mathrm{Al}$ XII & $1 s 5 d{ }^{1} D_{2}$ & $\left\{\begin{array}{l}\left(1 s 5 d^{1} D_{2}-1 s^{2}{ }^{1} S_{0}+\omega_{\text {las }}\right. \\
1 s 5 d{ }^{1} D_{2}-1 s^{2}{ }^{1} S_{0}-\omega_{\text {las }}\end{array}\right.$ & $\begin{array}{l}6.1712(8) \\
6.1789(8)\end{array}$ & $1.6194(3) \times 10^{7}$ & $1.6192 \times 10^{7}$ \\
\hline
\end{tabular}

Note. The experimental value for an ion in a laser field was determined from the equation $E\left[\mathrm{~cm}^{-1}\right]=10^{8}\left(1 / \lambda_{s 1}[\AA]+1 / \lambda_{s 2}[\AA]\right) / 2$; the calculation in Ref. 7 was performed for an isolated ion. 
resolution of the $\mathrm{x}$-ray spectrograph and especially increasing the dynamic range of the detection system will make it possible in the future to investigate weaker, higher-order nonlinear processes. ${ }^{11}$

The investigation of laser satellites is important for a number of reasons. First, the observation of laser satellites makes it possible to perform direct measurements of the excitation energies of the metastable states of ions and it can also be used to measure the intensities of laser fields in a plasma. Second, since the laser satellites are emitted only during the interaction with the heating laser pulse, they can be used to produce a source of ultrashort x-ray pulses. Finally, in analyzing x-ray laser schemes in which the upper level of the laser transition is metastable (for example, lasers based on $\mathrm{Ne}$ - and Ni-like ions), one must allow for processes that lead to the generation of laser satellites. ${ }^{3}$

We thank I. L. Berggman for helpful discussions. This work was supported in part by the Russian Fund for Fundamental Research, Grant No. 96-02-16111.

\footnotetext{
${ }^{1}$ A. V. Vinogradov and E. A. Yukov, Kvantovaya Élektron. (Moscow), No. 2, 105 (1973) [Sov. J. Quantum Electron. 3, 163 (1973)]

${ }^{2}$ I. Yu. Skobelev, A. Ya. Faenov, A. I. Magunov et al., Seventh International Conference on Multiphoton Processes, Garmisch-Partenkirchen, Germany, September 30-October 4, 1996; publ. Institute of Physics Conference Proceedings 154, edited by P. Lambropoulos and H. Walther.

${ }^{3}$ A. Osterheld, B. K. F. Young, J. Dunn et al., J. Quant. Spectrosc. Radiat. Transf. (1997), in press.

${ }^{4}$ H. R. Griem, R. C. Elton, and B. L. Welc, Seventh International Workshop on Radiative Properties of Hot Dense Matter, Santa Barbara, California, November 4-8, 1996.

${ }^{5}$ D. Strickland and G. Mourou, Opt. Commun. 56, 219 (1985); P. Maine, D. Strickland, P. Bado et al., IEEE J. Quantum Electron. QE-24, 398 (1988).

${ }^{6}$ I. Yu. Skobelev, A. Ya. Faenov, B. A. Bryunetkin et al., Zh. Éksp. Teor. Fiz. 108, 1263 (1995) [JETP 81, 692 (1995)].

${ }^{7}$ V. A. Bŏ̌ko, V. G. Pal'chikov, I. Yu. Skobelev, and A. Ya. Faenov, Spectroscopic Reference Data for Atoms and Ions [in Russian], Izd. Standartov, Moscow, 1988.

${ }^{8}$ L. A. Vaĭnshteĭn, I. I. Sobel'man, and E. A. Yukov, Excitation of Atoms and Spectral Line Broadening [in Russian], Nauka, Moscow, 1979.

${ }^{9}$ R. A. Mewe, Astron. Astrophys. 136, 906 (1962).

${ }^{10}$ H. Griem, Spectral Line Broadening by Plasmas, Academic Press, New York, 1974 [Russian translation, Mir, Moscow, 1978, p. 52].

${ }^{11}$ V. P. Gavrilenko and E. A. Oks, Kvantovaya Élektron. (Moscow) 10, 1910 (1983) [Sov. J. Quantum Electron. 13, 1269 (1983)].
}

Translated by M. E. Alferieff 